\title{
Les prérogatives de l'affectataire cultuel : étendue et limites
}

Frédéric Dieu

\section{OpenEdition}

1 Journals

Édition électronique

URL : http://journals.openedition.org/rdr/950

DOI : $10.4000 /$ rdr.950

ISSN : 2534-7462

Éditeur

Presses universitaires de Strasbourg

\section{Édition imprimée}

Date de publication : 8 novembre 2016

Pagination : 139-156

ISBN : 978-2-86820-959-7

ISSN : 2493-8637

\section{Référence électronique}

Frédéric Dieu, «Les prérogatives de l'affectataire cultuel : étendue et limites », Revue du droit des religions [En ligne], 2 | 2016, mis en ligne le 11 février 2020, consulté le 19 novembre 2020. URL : http:// journals.openedition.org/rdr/950; DOI : https://doi.org/10.4000/rdr.950

\section{(a) $(1)(9)$}

La revue du droit des religions est mise à disposition selon les termes de la Creative Commons Attribution - Pas d'Utilisation Commerciale 4.0 International - CC BY-NC 4.0. 


\section{LES PRÉROGATIVES DE L'AFFECTATAIRE CULTUEL : ÉTENDUE ET LIMITES}

\section{Frédéric DIEU}

Maître des requêtes au Conseil d'État

\section{RÉSUMÉ}

Le droit commun confère un large pouvoir d'utilisation du domaine public à la personne publique propriétaire. À l'inverse, il encadre strictement les droits de son occupant qui, précisément, ne l'occupe qu'à titre précaire. Il en va différemment en ce qui concerne le domaine public cultuel, c'est-à-dire l'ensemble des édifices du culte construits avant la loi de 1905 de séparation des Églises et de l'État. Pour ces édifices, en vue de garantir le libre exercice du culte, il est largement dérogé au droit commun en confiant à leur affectataire des prérogatives et des garanties qui vont bien au-delà des droits qui peuvent être conférés à l'occupant du domaine public. C'est à la nature et à l'étendue de ces prérogatives et garanties qu'est consacré cet article, qui tâchera d'indiquer dans quelle mesure le propriétaire public peut utiliser ces édifices à ses propres fins.

\section{Abstract}

The common law of public ownership provides the public authorities and local government with wide powers to use their public domain. In contrast, it strictly limits the rights of the occupant whose occupancy, precisely, is precarious. The situation is quite different as regards church buildings built before the 1905 Act of Separation, namely worship buildings belonging to public entities (mostly, towns and the State). In order to ensure freedom of worship, religious authority enjoys prerogatives and rights which go far beyond those granted to the occupant of ordinary buildings. This article focuses on the nature and extent of these rights. It also endeavours to show how the public owner of church buildings may use them for its own ends. 
Si la République ne reconnaît, ne salarie ni ne subventionne aucun culte, $\checkmark$ elle en garantit en revanche le libre exercice et accorde à cette fin aux différentes autorités religieuses la jouissance quasi exclusive des édifices du culte. Dans ce cadre, l'affectation cultuelle de ces édifices ainsi que les prérogatives du ministre du culte affectataire de cet édifice sont affirmées tant par la loi du 9 décembre 1905 de séparation des Églises et de l'État que par l'article L. 2124-31 du Code général de la propriété des personnes publiques qui consacre l'existence d'un domaine public cultuel s'agissant des édifices (les plus nombreux) construits avant 1905 et propriété des communes ou de l'État, tout en autorisant une utilisation de ces édifices à des fins non cultuelles, qu'il s'agisse d'en ouvrir l'accès pour des visites publiques ou d'y organiser des activités culturelles et même économiques (installation d'antennes-relais).

L'affectataire cultuel est réellement au centre de ce système, étant souverain pour tout ce qui concerne l'organisation du culte et disposant d'un « droit de veto » à l'égard de toute utilisation à des fins non cultuelles de l'édifice dont il a la charge. Il s'agit là de la meilleure garantie du respect de la liberté de culte qui a, on le sait, le caractère d'une liberté fondamentale.

L'on verra néanmoins que la forme (unilatérale ou contractuelle) que doit prendre ce nécessaire consentement de l'affectataire cultuel n'est pas imposée par les textes et que la jurisprudence a en outre affirmé la possibilité pour le propriétaire de l'édifice de l'utiliser à des fins non cultuelles sans avoir besoin de recueillir ce consentement. Mais il semble bien que cette possibilité demeure l'exception.

\section{QUELLES SONT LA SOURCE ET LA NATURE DE L'AFFECTATION CULTUELLE?}

\subsection{L'AFFECTATION CULTUELLE TROUVE SA SOURCE DANS LA LOI DE SÉPARATION}

On sait que les édifices du culte et leurs dépendances immobilières construits avant 1905 (nous ne pousserons pas le raffinement, dans cet article, jusqu'à distinguer, parmi eux, ceux construits avant le Concordat de 1801) «sont et demeurent propriétés de l'État, des départements, des communes et des établissements publics de coopération intercommunale ayant pris la compétence en matière d'édifices du culte » (art. 12 de la loi du 9 déc. 1905). 
Le Conseil d'État a tôt jugé que ces édifices relevaient du domaine public de ces collectivités (les communes pour les églises et l'État, en général, pour les cathédrales) et qu'il leur appartenait seules d'y entreprendre les travaux de gros entretien ${ }^{1}$. L'article 13 de la même loi prévoit que « les édifices servant à l'exercice public du culte, ainsi que les objets mobiliers les garnissant, seront laissés gratuitement à la disposition des établissements publics du culte * tandis que l'article 5 de la loi du 2 janvier 1907 est venu préciser pour le culte catholique (qui refusait la constitution d'associations cultuelles) que ces édifices « continueront à être laissés à la disposition des fidèles et des ministres du culte pour la pratique de leur religion $»$.

Ce sont donc ces dispositions législatives qui garantissent l'existence du domaine public cultuel (l'existence de ce domaine public étant confirmée et consacrée par la présence d'une disposition spécifique dans le Code général de la propriété des personnes publiques : art. L. 2124-31) et de l'affectation au culte, laquelle est réputée gratuite, perpétuelle et totale. Dans l'une des cinq décisions d'assemblée du 19 juillet 2011, le Conseil d'État, après avoir cité les dispositions de l'article 13 de la loi de 1905, a ainsi solennellement réaffirmé que « les dispositions de l'article 5 de la loi du 2 janvier 1907 garantissent, même en l'absence d'associations cultuelles, un droit de jouissance exclusive, libre et gratuite des édifices cultuels qui appartiennent à des collectivités publiques, au profit des fidèles et des ministres du culte, ces derniers étant chargés de régler l'usage de ces édifices, de manière à assurer aux fidèles la pratique de leur religion $»^{2}$. C'est donc dans la loi de séparation des Églises et de l'État que l'affectation cultuelle trouve sa source.

\section{2. unE AFFECTATION CULTUELLE GRATUIte, PERPÉtUELLE ET TOTALE...}

Arrêtons-nous sur le caractère perpétuel et total de l'affectation cultuelle, le caractère gratuit de cette affectation ne nécessitant pas de longs développements. Si ce caractère gratuit s'accompagne très généralement de la gratuité de l'accès à l'édifice cultuel, il ne prive pas l'affectataire cultuel de la possibilité de rendre payante la visite de parties de cet édifice affectées au culte, ainsi que le prévoit expressément l'article L. 2124-31 du Code général de la propriété des personnes publiques sur lequel nous reviendrons. Relevons à cet égard que le second alinéa de cet article précise que ce droit d'entrée, qui a le caractère d'une redevance domaniale, «peut être

1. CE, 10 juin 1921, Commune de Montségur, Rec. CE, p. 573.

2. CE, ass., 19 juill. 2011, n 308544, Commune de Trélazé. 
partagé entre la collectivité propriétaire et l'affectataire », ce qui signifie qu'il n'a pas forcément à l'être et qu'il peut être perçu, nous semble-t-il, par le seul propriétaire. Mais l'on peut penser que l'affectataire cultuel hésiterait à donner son accord à l'organisation de visites donnant lieu à la perception d'un droit d'entrée qui ne profiterait qu'à la commune ou à l'État propriétaires de l'édifice cultuel.

Le caractère perpétuel de l'affectation cultuelle signifie en particulier que l'édifice est toujours maintenu à la disposition du culte affectataire, ce tant que sa désaffectation n'a pas été expressément prononcée en application de l'alinéa 2 de l'article 13 de la loi de 1905 (lequel distingue cinq cas de désaffectation par arrêté préfectoral, notamment : dissolution de l'association cultuelle, absence de culte pendant plus de six mois consécutifs, conservation de l'édifice compromise par l'insuffisance d'entretien, édifices détournés de leur destination ou association cessant de remplir son objet) : c'est pourquoi le non-usage cultuel de l'édifice ne saurait lui ôter la perpétuité de son affectation cultuelle, de sorte qu'il n'existe, pour le domaine public du culte, aucune désaffectation de fait, et que le juge des référés du Conseil d'État a pu relever et sanctionner une atteinte grave à la liberté fondamentale du culte « alors même qu'aucune célébration d'un office religieux n'était prévue aux dates fixées pour les manifestations autorisées [pièce de théâtre, conférences, exposition de peinture, concert] $»^{3}$. Il a de même été jugé que l'affectation cultuelle perdurait même en cas de démolition de l'édifice ${ }^{4}$, d'utilisation profane, occasionnellement, de l'édifice ${ }^{5}$ et d'absence de célébration du culte $e^{6}$. La désaffectation cultuelle ne peut en conséquence intervenir que dans les conditions prévues par l'article 13 de la loi de $1905^{7}$, ce qui, en l'état actuel des textes (décret $n^{\circ} 70-220$ du 17 mars 1970), impose de procéder par arrêté préfectoral pris à la demande du conseil municipal et fondé sur le consentement écrit de la personne qualifiée pour représenter le culte affectataire.

Le caractère total de l'affectation cultuelle signifie quant à lui que c'est en principe l'ensemble de l'édifice, dans toute sa superficie, qui est destiné

3. CE, ord. réf., 25 août 2005, n 284307, Commune de Massat, Rec. CE, p. 386.

4. CE, 18 mars 1988, n 44141, Maron, JCP G 1988, II, nº 21192.

5. CAA Bordeaux, 27 avr. 2004, Association Église Saint-Éloi, JCP A, 17 mai 2004, note TAWIL E.

6. CE, 30 déc. 2002, n 248787, Commune de Pont-Audemer.

7. CE, $1^{\text {er }}$ avril 1938, Abbé Laplanche-Coudert, Rec. CE, p. 339 : décision affirmant l'affectation cultuelle du calvaire situé sur la place communale qui constitue le point d'arrivée des processions de la Fête-Dieu. 
et affecté au culte et uniquement ou du moins prioritairement à cela : ce sont ainsi l'ensemble des dépendances immobilières de l'édifice et des objets mobiliers le garnissant et nécessaires à l'exercice du culte qui bénéficient de l'affectation cultuelle et la jurisprudence a souvent retenu une conception extensive de cette affectation. Le Conseil d'État a ainsi jugé (ce qui n'est certes guère surprenant) que les objets mobiliers nécessaires à l'office divin (chrétien) comme les croix étaient affectés au culte ${ }^{8}$ et affirmé (ce qui était moins évident) qu'une sacristie accolée à la façade d'une église " constituait, au même titre que les autres parties de l'église, une dépendance du domaine public communal [affectée au culte] $»^{9}$ et que des locaux commerciaux situés au-dessous d'une chapelle (la chapelle absidiale de l'église Saint-Eustache à Paris) étaient affectés au culte dès lors qu'ils faisaient corps avec les parties de l'édifice servant directement à l'exercice du culte ${ }^{10}$.

Dans la décision Maron ${ }^{11}$, le Conseil d'État avait fondé l'affectation cultuelle sur la solidarité matérielle, physique, entre la dépendance en cause et l'édifice du culte. La décision Commune des Saintes-Maries-de-la-Mer est revenue sur cette jurisprudence en jugeant que l'affectation au culte concerne l'édifice et ses « dépendances nécessaires, fonctionnellement indissociables de l'édifice cultuel ${ }^{12}$. L'indissociabilité matérielle avec l'édifice ne suffit donc pas à caractériser l'affectation au culte, encore faut-il que la dépendance en cause ait bien une fonction cultuelle (soit, comme la toiture, "nécessaire au bon déroulement des célébrations cultuelles ») et/ou ne soit accessible qu'en empruntant les «parties internes» de l'édifice.

\section{3. ... MAIS NON EXCLUSIVE}

Enfin, pour être entière, l'affectation cultuelle n'en est cependant pas exclusive : en effet, les dispositions de la loi de 1905 ne font par ellesmêmes pas obstacle à un usage non cultuel (notamment culturel) de l'édifice cultuel, usage qui serait décidé par le propriétaire de l'édifice, soit, le plus souvent, la commune. La loi de 1905 s'appliquant aux « édifices servant à l'exercice public du culte» (art. 13), lesquels sont laissés à la

8. Ibid.

9. CE, $1^{\text {er }}$ août 1919, Abbé Lachaud, Rec. CE, p. 698 ; 18 mars 1988, Maron, JCP G 1988, II, n 21192. - V. aussi CE, 18 nov. 1949, Sieur Carlier, Rec. CE, p. 490 : affectation cultuelle de parties de l'édifice accessibles aux seuls desservants.

10. TA Paris, 8 juin 1971, AJDA 1972, p. 169.

11. CE, 18 mars 1988, précit.

12. CE, 20 juin 2012, n 340648, Commune des Saintes-Maries-de-la-Mer, Rec. CE, p. 247. 
disposition d'associations cultuelles qui « devront avoir exclusivement pour objet l'exercice d'un culte» (art. 19), il en résulte que la jouissance des édifices cultuels est accordée aux associations cultuelles (ou diocésaines) dans un but unique, l'exercice de leur culte, qui est exclusif de tout autre, l'article 5 de la loi du 2 janvier 1907 indiquant également que les édifices sont laissés à la disposition des ministres du culte "pour la pratique de leur religion».

La même loi prévoit la visite des édifices cultuels, notamment ceux qui sont classés monuments historiques - ils sont nombreux - ou qui abritent des objets mobiliers classés ou inscrits. Aux termes de l'article 17, in fine, de cette loi : « la visite des édifices et l'exposition des objets mobiliers classés seront publiques: elles ne pourront donner lieu à aucune taxe ni redevance ». On sait que l'article L. 2124-31 du Code général de la propriété des personnes publiques est revenu sur cette gratuité obligatoire, en prévoyant la possibilité de percevoir une redevance domaniale.

Un arrêt de la deuxième chambre civile de la Cour de cassation a synthétisé cette exclusivité de l'usage cultuel en jugeant que le pouvoir de police du desservant «n'a pour but que de lui permettre d'assurer l'exercice du culte », la Cour ajoutant, pour illustrer son propos, qu'à ce titre, le desservant « est en droit de fixer les horaires des services religieux, d'ordonner les cérémonies, et de faire expulser les perturbateurs, et qu'en contrepartie de ce pouvoir sacerdotal, il a l'obligation de respecter le libre droit des fidèles de pénétrer dans l'église et de participer au culte ${ }^{13}$.

Autrement dit, et c'est essentiel, l'exclusivité de l'affectation cultuelle est une obligation pesant sur l'affectataire de l'édifice cultuel, c'est-à-dire l'association cultuelle et son desservant, et non sur le propriétaire de cet édifice, c'est-à-dire la personne publique (commune ou État en général). Cette dernière peut en conséquence décider d'un usage non cultuel de l'édifice cultuel. La question essentielle n'est donc pas celle de la possibilité d'un tel usage, mais celle de ses modalités, en particulier de son initiative, unilatérale (décision de la personne publique) ou concertée (accord du desservant).

13. Cass. $2^{e}$ civ., 19 juill. 1966, Vautier, Bull. crim., nº 182 ; JCP G 1967, II, nº 15228. 


\section{IDENTITÉ ET POUVOIRS DE L'AFFECTATAIRE CULTUEL}

\subsection{LA DÉTERMINATION DE L'IDENTITÉ DE L'AFFECTATAIRE CULTUEL}

Il résulte de la jurisprudence que, pour le juge « civil » ou étatique (qu'il soit administratif ou judiciaire), c'est-à-dire le juge qui demeure extérieur et étranger à l'organisation interne du culte en cause, l'affectataire cultuel est le ministre du culte reconnu et désigné comme tel par la hiérarchie propre à son culte. Sur ce point, le juge civil se borne à prendre acte de la décision et de la position de la hiérarchie religieuse ${ }^{14}$. Ainsi, pour l'Église catholique, l'affectataire est le curé desservant l'église (ou les églises) de la paroisse, nommé par l'évêque du diocèse territorialement compétent. Plus généralement, ainsi que l'indique la circulaire du 29 juillet 2011 relative aux édifices du culte (§ 1.3), l'affectataire légitime de l'édifice est « le desservant régulièrement nommé par les autorités de son culte et en communion avec la hiérarchie de celui-ci ». La jurisprudence illustre très bien l'obligation dans laquelle se trouve l'administration de se conformer à l'organisation et aux décisions des autorités religieuses.

Ainsi, s'agissant de la Guyane, où le statut des Églises demeure régi par une ordonnance du 27 août 1828 et où les ministres du culte catholique sont payés par le département et agréés par le préfet, le Conseil d'État a jugé que le préfet était tenu de radier des cadres le ministre du culte catholique qui avait fait l'objet, de la part de l'évêque de Cayenne, « autorité représentative de l'Église catholique en Guyane », d'une décision de mutation en Haïti ${ }^{15}$. De même, s'agissant de l'Alsace-Moselle, le Conseil d'État a jugé que l'autorité administrative dans ces départements était tenue de radier et de cesser de rémunérer un ministre du culte catholique qui avait été révoqué par l'évêque de Strasbourg pour rupture avec la communauté diocésaine ${ }^{16}$.

Ce « légitimisme religieux » de l'autorité administrative et du juge étatique s'est également manifesté à propos des conflits entre prêtres pour l'occupation

14. Ainsi que l'indiquait D. LABETOulle dans ses conclusions sous la décision de section du 17 octobre 1980 (Pont, Rec. CE, p. 374) : « [...] la décision de l'Église est, pour l'administration, une donnée de fait et non de droit [...] Et il n'appartient à aucune juridiction étatique ni administrative ni judiciaire d'apprécier la conformité de cette décision aux règles internes de cette Église. » L'esprit de la loi de 1905 en particulier est que « l'État se borne à prendre acte de la façon dont, sur ce point, l'Église se présente à lui » (ibid.).

15. CE, 9 oct. 1981, n 18649, Beherec, Rec. CE, p. 358.

16. CE, 2 déc. 1981, n 27903, Ministre de l'Intérieur c/ Siegel, Rec. CE, tables, p. 739. 
d'une église. Ainsi, par un célèbre arrêt du 6 février $1912^{17}$, la chambre civile de la Cour de cassation a jugé que l'attribution de l'église devait être dans un tel cas « exclusivement réservée » à celui des deux prêtres qui « se soumet aux règles d'organisation générale du culte dont il se propose d'assurer l'exercice, notamment à celle de la hiérarchie ecclésiastique qui demeure en communion avec son évêque ». Le Conseil d'État a de même annulé une décision attribuant la jouissance d'une église communale à un prêtre interdit par son évêque et refusant de céder "sa place » au prêtre désigné par ce même évêque ${ }^{18}$, tandis que le tribunal administratif d'Amiens a annulé un arrêté municipal confiant à une association non reconnue par l'évêque du lieu le gardiennage et l'entretien d'une église ${ }^{19}$. Plus récemment, le Conseil d'État a dénié la compétence du juge administratif pour statuer sur la contestation de la décision de nomination du curé titulaire d'une paroisse prise par un évêque ${ }^{20}$.

\subsection{LES PRÉROGATIVES DE L'AFFECTATAIRE CULTUEL : DES POUVOIRS « FINALISÉS ॥}

Les dispositions de la loi de Séparation ont été interprétées par le juge administratif comme confiant au seul ministre du culte desservant l'édifice cultuel le soin d'en régler l'usage afin, bien sûr, d'organiser l'exercice du culte : il a, selon la décision de section Abbé Tisseire du 26 décembre $1930^{21}$, « la garde et la police de l'église en vue d'assurer aux fidèles l'exercice de leur religion ». L'affectataire dispose ainsi, seul, d'un pouvoir d'organisation et de police du culte à l'intérieur de l'édifice cultuel qui lui est affecté. Le maire, qui est pourtant l'exécutif de la collectivité propriétaire de l'édifice, est ainsi privé de tous pouvoirs à l'intérieur de l'édifice cultuel (construit avant 1905), sauf s'il s'agit d'assurer le respect de l'ordre public, notamment de la sécurité publique ${ }^{22}$ ou de régler les sonneries civiles sur le fondement

17. Cass. civ, 6 févr. 1912, D. 1912, p. 121 ; S. 1913, p. 137, note Mestre A. : en l'espèce, l'affectation au culte catholique a été admise pour «l'exercice d'un culte pratiqué selon les préceptes de l'Église catholique par des prêtres reconnaissant la hiérarchie et faisant partie de cette hiérarchie ». - V. aussi CA Paris, 13 juin 1957, D. 1957, somm. p. 145.

18. CE, 23 janv. 1920, Abbé Barraud, Rec. CE, p. 75 ; 28 juill. 1911, Rougegré et autres, Rec. CE, p. 909.

19. TA Amiens, 16 sept. 1986, Mgr Mabille, Rec. CE, p. 320 : jugement relevant que l'association traditionaliste en cause n'observe pas le principe de "soumission à la hiérarchie de l'Église catholique ".

20. CE, 17 oct. 2012, n $\mathrm{n}^{\circ} 352742$, M. Singa.

21. CE, sect., 26 déc. 1930, Abbé Tisseire, Rec. CE, p. 1114.

22. CE, 26 mai 1911, Ferry, Rec. CE, p. 638 : menace d'effondrement de l'église. V. aussi CE, 26 déc. 1913, Abbé Lhuillier curé de Saint-Paterne à Orléans, Rec. CE, p. 1295. 
de l'article 27 de la loi de $1905^{23}$. Le maire ne peut donc, par exemple, désigner un gardien « civil» qui serait chargé d'organiser des visites dans l'église ${ }^{24}$ ou, plus généralement s'immiscer dans la police du lieu de culte ${ }^{25}$. C'est donc tout l'ordre intérieur qui relève du desservant et c'est l'ensemble de l'intérieur de l'édifice qui est sous sa seule responsabilité, le Conseil d'État s'étant de longue date refusé à diviser l'espace intérieur de l'édifice cultuel pour y appliquer des régimes différents ${ }^{26}$.

Concrètement, c'est donc au seul desservant qu'il appartient de fixer les horaires des célébrations et plus largement les horaires d'ouverture et de fermeture de l'édifice ${ }^{27}$, d'organiser les cérémonies religieuses ${ }^{28}$, d'assurer la police intérieure de l'édifice ${ }^{29}$ et plus généralement de régler l'utilisation de l'édifice et du mobilier qui la garnit ainsi que l'aménagement intérieur des lieux ${ }^{30}$. Lui seul est propriétaire de plein droit des clés de l'édifice ${ }^{31}$, alors même qu'il n'en est pas le propriétaire mais seulement l'affectataire. Beaucoup plus récemment, la cour administrative d'appel de Marseille a ainsi jugé que seul le ministre du culte était compétent pour autoriser la réalisation de travaux d'embellissement et de décoration à l'intérieur d'un édifice du culte ${ }^{32}$, ce qui avait pour effet de contraindre le maire à recueillir son accord avant de mettre à exécution de tels travaux.

Très protectrice de ce droit de jouissance exclusif de l'édifice cultuel du desservant, la jurisprudence du Conseil d'État a pu affirmer qu'aucune décision ne pouvait « avoir indirectement pour effet d'enlever au ministre du

23. CE, 24 févr. 1912, Abbé Sarralongue, Rec. CE, p. 250 ; 11 avril 1913, Abbé Sommé, Rec. CE, p. 392.

24. CE, 3 mai 1918, Abbé Piat, Rec. CE, p. 409.

25. Décision Abbé Sarralongue, précit.

26. CE, sect., 15 juill. 1938, Association cultuelle d'Allondans-Dung, Rec. CE, p. 673.

27. Décisions Abbé Sarralongue et Abbé Sommé, précit.

28. Cass. crim., $1^{\text {er }}$ déc. 1910, D. 1911, I, p. 351.

29. Décision Abbé Piat, précit.

30. CE, 17 déc. 1914, Abbé Foussadier, Rec. CE, p. 1052 ; 4 août 1916, Abbé Prudhommeaux, Rec. CE, p. 354 ; décision Abbé Tisseire, précit.

31. Décision Abbé Sommé, précit. ; CE, 20 juin 1913, Abbé Arnaud, Rec. CE, p. 717 : illégalité d'un arrêté municipal prescrivant de déposer à la mairie une clé de la porte de l'église, car cette prescription a pour conséquence de permettre au maire d'entrer dans l'église en dehors des horaires fixés par le ministre du culte affectataire.

32. CAA Marseille, 22 nov. 2011, n 10MA00428, Commune de Saint-Étienne-du-Grès, AJDA 2012, p. 497, concl. Deliancourt S. : « la réalisation de la fresque n'avait pas le caractère de travaux nécessaires à la conservation et à l'entretien de l'édifice, contrairement à ce que persiste à soutenir en appel la commune, mais une œuvre décorative, laquelle relevait de la seule compétence du ministre du culte en charge de la garde et de la police de ladite chapelle. » 
culte occupant l'église le droit d'en régler l'usage ${ }^{33}$. Au total donc, l'affectataire cultuel est souverain en matière d'organisation du culte à l'intérieur de l'édifice cultuel : ni le propriétaire de l'édifice (la commune en général), ni l'« usager» du culte (le paroissien pour les Églises chrétiennes) ne peuvent contester ses décisions devant le juge civil : seules l'autorité religieuse et la juridiction propre à la religion concernée sont à cet égard compétentes pour trancher un conflit.

L'exclusivité ainsi accordée à l'affectataire cultuel dans l'organisation du culte au sein de l'édifice cultuel, et l'étendue des pouvoirs qui lui sont accordés dans ce cadre, sont, si l'on peut dire, "finalisés ", c'est-à-dire affirmés et garantis afin de «faire respecter la destination cultuelle de l'édifice, voulue par la loi, et de réglementer en conséquence l'utilisation de l'église et de son mobilier ${ }^{34}$. La première fonction de l'affectataire cultuel est donc « la protection de la destination cultuelle ${ }^{35}$, ce pourquoi il ne peut lui être imposé " aucune obligation de caractère matériel à l'intérieur de l'église, telle qu'une obligation de sécurité qui tendrait à l'assimiler à l'exploitant d'un lieu ouvert au public ou à une collectivité publique administrant un bien du domaine public ${ }^{36}$.

Cependant, l'affectation au culte se faisant par détermination de la loi, l'autorité religieuse n'est pas compétente pour affecter ou désaffecter un édifice ${ }^{37}$. Par ailleurs, et toujours en vertu de dispositions législatives, l'affectataire ne peut tenir de réunions politiques dans l'édifice qui lui est confié (art. 26 de la loi du 9 décembre 1905) et il ne peut y prononcer un discours ou y afficher ou y distribuer un écrit contenant des propos outrageants ou diffamatoires à l'égard d'un citoyen chargé d'un service public ou incitant les citoyens à résister à l'exécution des lois ou encore dressant les citoyens les uns contre les autres (art. 34 et 35).

33. Décision Abbé Arnaud, précit.

34. Gaudemet Y., Traité de droit administratif. Droit administratif des biens, t. 2, Paris, LGDJ, $14^{\mathrm{e}}$ éd. 2012, p. 107.

35. Délicat Y., « Les pouvoirs de l'affectataire cultuel », AJDA, 2012, p. 2103.

36. Cass. $2^{e}$ civ., 19 juill. 1966, SNCF et Dame Vautier c/ Chanoine Rebuffat, Bull civ., nº 812 ; V. sur ce point $\& 1.4$ de la circulaire précitée du 29 juillet 2011.

37. CE, $1^{\text {er }}$ mars 1912, Commune de Saint-Dezery, Rec. CE, p. 299 : incompétence pour affecter ; CE, 15 juill. 1938, Association cultuelle d'Allondans-Dung et Consistoire de Montbéliard, Rec. CE, p. 673 : incompétence pour désaffecter. 


\section{QUAND L'ACCORD DE L'AFFECTATAIRE EST-IL REQUIS ET QUAND NE L'EST-IL PAS?}

\subsection{L'ACCORD DE L'AFFECTATAIRE EST NÉCESSAIRE À L'ÉGARD DE L'UTILISATION DE L'ÉDIFICE CULTUEL ET DE CEUX DE SES AMÉNAGEMENTS, ÉLÉMENTS ET DÉPENDANCES QUI EN SONT FONCTIONNELLEMENT INDISSOCIABLES}

À titre liminaire, puisque l'on vient d'évoquer l'incompétence de l'autorité cultuelle pour affecter au culte ou désaffecter un édifice, l'on doit immédiatement préciser que le consentement de la personne physique ou morale pour représenter le culte affectataire est requis pour qu'il soit procédé à la désaffectation de l'édifice, ce en application des dispositions de l'article 13 de la loi de 1905 (et de l'article 5 de la loi du 2 janvier 1907 pour le culte catholique) et de l'article $1^{\text {er }}$ du décret du 17 mars 1970. En conséquence, est illégale la délibération d'un conseil municipal autorisant une désaffectation sans avoir recueilli le consentement du représentant du culte affectataire ou mettant l'édifice cultuel à la disposition d'une association sans que celui-ci ait donné son accord ${ }^{38}$.

Par ailleurs, outre «la visite de parties d'édifices affectés au culte, notamment de celles où sont exposés des objets mobiliers classés ou inscrits », pour lesquelles l'accès à l'édifice " est subordonné à l'accord de l'affectataire », ce sont plus généralement toutes les utilisations de l'édifice cultuel à des fins non cultuelles qui sont, en vertu de l'article L. 2124-31 du Code général de la propriété des personnes publiques ${ }^{39}$, soumises au consentement de l'affectataire cultuel. Mais encore faut-il, précise cet article, que ces utilisations se rapportent à des "activités compatibles avec l'affectation cultuelle», ce qui semble vouloir dire que l'affectataire ne peut donner son accord à une utilisation de l'édifice incompatible avec cette affectation et qu'un tel accord, fût-il donné, serait sans effet. L'accord de l'affectataire doit préciser les « conditions et les modalités de cet accès [visite de l'édifice] ou de cette utilisation [utilisation de l'édifice pour une activité compatible avec l'affectation cultuelle]». L'article L. 2124-31 ne précise pas la forme que doit prendre cet accord : il nous semble qu'il peut être aussi bien unilatéral (décision de l'affectataire) que contractuel (convention conclue par l'affectataire et le propriétaire, généralement la

38. CAA Bordeaux, Association Église Saint-Éloi, précit.

39. Qui est une confirmation législative de la jurisprudence administrative relative aux utilisations de l'édifice cultuel compatibles avec l'affectation cultuelle. 
commune) mais, dès lors que l'accès ou l'utilisation doit donner lieu à la perception d'une redevance domaniale dont le produit est partagé par l'affectataire et le propriétaire, la voie contractuelle est certainement la plus adaptée.

La règle de l'accord obligatoire du desservant déroge, soulignons-le, au droit commun de la domanialité publique qui ne prévoit nullement que l'utilisation du domaine par la personne publique propriétaire est subordonnée à l'accord de l'occupant du domaine et qui garantit au propriétaire un droit d'usage dès lors que l'utilisation du domaine revêt un caractère anormal, comme c'est le cas pour l'utilisation non cultuelle de l'édifice cultuel. C'est qu'en effet le desservant est plus qu'un simple occupant du domaine public : il en est l'affectataire, alors même que le droit commun de la domanialité publique ne connaît que l'affectation à l'usage du public ou au service public.

Jusqu'à la décision précitée Commune des Saintes-Maries-de-la-Mer, le Conseil d'État jugeait que toute utilisation non cultuelle de l'édifice cultuel ${ }^{40}$ ne pouvait être décidée par la collectivité propriétaire de l'édifice qu'après que celle-ci eût recueilli l'accord du desservant. Cette décision est venue quelque peu limiter cette obligation en prévoyant qu'elle ne s'applique qu'à l'utilisation des dépendances, éléments et aménagements indissociables de l'édifice cultuel. Dès 1931, le Conseil d'État avait jugé que le maire ne pouvait prescrire l'utilisation de l'église communale en vue d'une fin non cultuelle, alors même qu'il n'existait aucune incompatibilité entre l'usage ainsi prescrit par le maire et l'affectation au culte ${ }^{41}$. Par une décision de section du 4 novembre $1994^{42}$, dans laquelle était en cause la décision d'un maire d'instituer un droit de visite d'objets mobiliers exposés dans l'église, le Conseil d'État a ainsi jugé que, même lorsque les objets en cause sont classés, de telles visites ne peuvent être organisées qu'avec l'accord du desservant et qu'à défaut d'un tel accord, la délibération du conseil municipal «porte atteinte aux droits qui sont reconnus à ce dernier pour réglementer l'usage des biens laissés à la disposition des fidèles par les lois des 9 décembre 1905 et 2 janvier 1907 ». Il serait aujourd'hui jugé qu'une telle délibération méconnaît également les

40. Il faut cependant réserver l'hypothèse d'une désaffectation formelle et expresse de l'édifice cultuel qui autorise ensuite son utilisation culturelle sur seule décision de la commune : CE, 21 juin 1989, n 73108, Gindre : affectation d'une ancienne église à un service public à caractère culturel.

41. CE, 9 janv. 1931, Abbé Cadel, S. 1931, III, p. 41.

42. CE, 4 nov. 1994, n 135842, Abbé Chalumey, Rec. CE, p. 491 ; RFDA 1995, p. 986, concl. SCHWARTZ R. 
dispositions de l'article L. 2124-31 du Code général de la propriété des personnes publiques.

La nécessité d'un accord du ministre du culte a été confirmée par une ordonnance du 25 août $2005^{43}$ dans laquelle le juge des référés du Conseil d'État a affirmé que le maire d'une commune « commet une illégalité manifeste en autorisant une manifestation dans un édifice affecté à l'exercice d'un culte sans l'accord du ministre du culte chargé d'en régler l'usage " : dans cette affaire, le maire était même passé outre «l'opposition motivée » du ministre du culte et avait été retenue une atteinte grave à la liberté fondamentale du culte « alors même qu'aucune célébration d'un office religieux n'était prévue aux dates fixées pour les manifestations autorisées ». Plus récemment, il a été jugé que l'accord de l'affectataire était nécessaire pour l'installation dans une église d'un orgue acquis par la commune propriétaire de l'édifice « afin notamment de développer l'enseignement artistique et d'organiser des manifestations culturelles ${ }^{44}$ : dans cette hypothèse, c'est la nécessité de « garantir une utilisation de l'orgue par la commune conforme à ses besoins et une participation de l'affectataire ou du propriétaire de l'édifice, dont le montant soit proportionné à l'utilisation qu'il pourra faire de l'orgue », qui impose un consentement exprès de l'affectataire, consentement qui prendra vraisemblablement la forme d'une convention avec la commune propriétaire ainsi que le Conseil d'État l'a suggéré dans la décision précitée Commune de Trélazé, laquelle fixera en particulier les engagements réciproques de l'affectataire et du propriétaire.

La nécessité pour la commune de recueillir l'accord de l'affectataire cultuel s'impose également lorsque celle-ci souhaite utiliser l'édifice non pas pour ses propres « besoins culturels» mais pour les besoins techniques et économiques d'un tiers : tel est le cas pour la pratique, qui semble se développer, de l'installation d'antennes-relais de téléphonie mobile dans les clochers des églises, la commune propriétaire devant alors rechercher l'accord de l'affectataire en vue de permettre à l'opérateur de téléphonie d'installer ses antennes dès lors que le clocher est une partie de l'édifice affectée au culte ${ }^{45}$. La cour

43. Décision Commune de Massat, précit., AJDA 2006, p. 91, note Subra DE Bieusses P.

44. Décision Commune de Trélazé, précit.

45. L'article 51 du décret du 16 mars 1906 indique que les cloches « servent à l'exercice public du culte » tandis que l'article 52 précise qu'une clé du clocher est déposée entre les mains de l'affectataire, une autre dans les mains du maire qui « ne peut en faire usage que pour les sonneries civiles $»$. Pour un exemple de sonneries civiles excessives, V. CAA Paris, 5 nov. 2013, $\mathrm{n}^{\circ}$ 10PA04789, Commune de Boissette : annulation de la décision du maire refusant de mettre fin à l'utilisation des cloches de l'église à des fins civiles toutes 
administrative de Marseille a jugé à cet égard que l'installation d'antennesrelais dans le clocher d'une église était compatible avec l'affectation cultuelle de celui-ci, mais soumise à l'accord de l'affectataire dès lors que l'installation imposait un accès permanent au clocher et que celui-ci ne disposait pas « d'un accès direct sur l'extérieur distinct de celui utilisé par les fidèles pour pénétrer dans l'église ». L'affectataire n'ayant pas donné son accord, la cour a annulé, à la demande de ce dernier, la décision du maire refusant d'enlever l'installation ${ }^{46}$. La cour administrative d'appel de Nantes a retenu la même solution à l'encontre de la décision du maire, prise sans l'accord de l'affectataire, de délivrer un permis de construire à l'opérateur de téléphonie mobile, ce après avoir relevé que le clocher, "alors même qu'il aurait été inaccessible au public ${ }^{47}$, faisait partie intégrante de l'édifice, dont il n'est pas dissociable ${ }^{48}$. Ces arrêts soulèvent deux (au moins) questions.

La première porte sur la possibilité de faire application de la jurisprudence Commune des Saintes-Maries-de-la-Mer - dont nous avons vu qu'elle n'impose pas de recueillir l'accord de l'affectataire pour la visite des parties de l'édifice cultuel ayant un accès indépendant dépourvu de toute communication avec les parties internes de l'église - aux utilisations qui sont faites du clocher aux fins d'y installer des antennes-relais. Ce qui vaut pour les visites du public vaut-il pour des utilisations privatives par des tiers à des fins économiques? L'arrêt précité de la cour administrative de Marseille semble admettre que le consentement de l'affectataire ne sera pas requis si le clocher bénéficie (en bref) d'un accès indépendant de celui des fidèles tandis que celui de la cour administrative d'appel de Nantes semble exiger ce consentement dans tous les cas. Le Conseil d'État aura probablement l'occasion de clarifier ce point.

La seconde question porte plus radicalement sur la compatibilité entre l'installation d'antennes-relais et le culte, l'exigence de compatibilité entre l'activité prenant place dans l'édifice cultuel et l'affectation cultuelle étant posée par l'article L. 2124-31 CGPPP. Eu égard à son caractère privatif et à sa motivation économique ainsi qu'au fait qu'elle nécessite un accès permanent au clocher (et donc la délivrance d'une clé à l'opérateur), l'activité d'installation et d'exploitation d'antennes-relais n'apparaît pas avec évidence compatible

les heures et toutes les demi-heures deux fois de suite, de jour comme de nuit (arrêt faisant l'objet d'un pourvoi en cassation).

46. CAA Marseille, 4 oct. 2013, n 11MA04486, M. Gounelle.

47. Ce qui est le cas de la plupart des clochers d'églises.

48. CAA Nantes, 29 nov. 2013, n 12NT00939, Abbé Brindejonc et autres. Annulation en conséquence des permis de construire. 
avec l'affectation cultuelle, bien qu'un «intérêt public [...] s'attache à la couverture du territoire national par le réseau de téléphonie mobile ${ }^{49}$.

À cet égard, l'on peut s'interroger sur ce que pourraient faire tant l'opérateur que la commune en cas de désaccord de l'affectataire. A priori, un tel désaccord, qui n'a pas à être motivé, sera sans recours et il restera à la commune et à l'opérateur à jeter leur dévolu sur un autre clocher. Mais l'on peut (hélas) penser que certaines communes passeront outre, ce qui conduira l'affectataire à saisir le juge administratif (d'un référé-liberté - article L. 521-2 du Code de justice administrative ${ }^{50}$ - ou d'un référé-suspension - article L. 521-1 - accompagné d'un recours pour excès de pouvoir) de demandes tendant à mettre fin à l'installation. Dans l'hypothèse où l'affectataire aurait donné son accord mais où les modalités de cet accord ne seraient pas respectées par le propriétaire de l'édifice cultuel, et sans que cela porte atteinte à la liberté fondamentale de culte ${ }^{51}$, un référé «mesures utiles» pourrait également être présenté au juge administratif par l'affectataire. À l'inverse, dans l'hypothèse où l'affectataire a donné son accord, l'on ne peut exclure qu'un usager régulier de l'édifice (ou que l'autorité religieuse à laquelle est soumis l'affectataire) saisisse le juge administratif d'une demande identique afin de faire respecter l'affectation cultuelle du clocher.

\subsection{L'ACCORD DE L'AFFECTATAIRE N'EST PAS REQUIS À L'ÉGARD DE L'UTILISATION DES AMÉNAGEMENTS OU ÉLÉMENTS FONCTIONNELLEMENT DISSOCIABLES DE L'ÉDIFICE CULTUEL}

Telle est la solution résultant de la décision précitée Commune des SaintesMaries-de-la-Mer. En effet, si cette décision retient en principe une vision globale de l'édifice cultuel, l'analyse fonctionnelle des différentes parties de cet édifice à laquelle elle se livre conduit en fait à retenir une vision composite de l'édifice, les parties de l'édifice non affectées directement au culte étant disjointes de lui et pouvant en conséquence faire l'objet de visites payantes organisées par la commune propriétaire sans l'accord du desservant.

49. CE, 2 juill. 2008, $\mathrm{n}^{\circ} 310548$, Société française du radiotéléphone.

50. Nous avons vu en effet avec la décision précitée Commune de Massat que l'affectataire dont l'accord n'a pas été recueilli (ou dont le désaccord a été ignoré) au sujet d'une utilisation non cultuelle de l'édifice qui lui est confié peut saisir le juge administratif d'un référé-liberté destiné à mettre fin à l'atteinte grave et manifestement illégale portée à la liberté fondamentale qu'est la liberté de culte, laquelle a «pour composante la libre disposition des biens nécessaires à l'exercice d'un culte».

51. En présence d'une telle atteinte, c'est en effet le référé-liberté qui serait pertinent. 
Selon la décision en effet, l'affectation cultuelle « s'applique à l'ensemble d'un édifice cultuel, y compris ses dépendances nécessaires, fonctionnellement indissociables de l'édifice cultuel [et] la toiture d'un édifice cultuel, en tant qu'elle est nécessaire au bon déroulement des célébrations cultuelles organisées dans l'édifice qu'elle protège, est affectée au culte en vertu de ces mêmes dispositions ». Le Conseil reconnaît donc l'affectation cultuelle au toit de l'édifice, mais il la dénie à la terrasse située sur son dessus. En effet, la terrasse est à ranger parmi les aménagements "qui, alors même qu'ils sont situés sur le toit de l'édifice cultuel, doivent être regardés, compte tenu notamment de leurs caractéristiques propres et de la possibilité d'y accéder sans entrer dans l'édifice cultuel, comme fonctionnellement dissociables de cet édifice ». La circonstance que la terrasse et le chemin de ronde soient situés sur le toit de l'église, c'est-à-dire sur un immeuble affecté au culte, ne suffit donc pas à leur donner le caractère de « dépendances affectées au culte ». Autrement dit, l'indissociabilité physique et matérielle des dépendances et aménagements ne présume pas de leur indissociabilité fonctionnelle, c'est-à-dire de leur commune affectation à l'exercice du culte : la terrasse et le chemin de ronde ont beau être matériellement et physiquement indissociables de l'église et de son toit (lequel est nécessaire à l'exercice du culte puisqu'il assure la couverture de l'édifice), ils en sont fonctionnellement dissociables puisqu'ils ne sont pas eux-mêmes affectés à l'exercice du culte et sont accessibles par l'extérieur de l'édifice cultuel. Les fidèles pouvant accéder à l'église sans emprunter cette terrasse et ce chemin et les touristes pouvant, symétriquement, accéder à cette terrasse et ce chemin sans emprunter des édifices ou dépendances affectés au culte, la séparation fonctionnelle est totale et vient occulter la solidarité physique et matérielle. La terrasse et le chemin de ronde ne sont pas affectés au culte : or, c'est seulement s'ils l'étaient que leur visite devrait être autorisée par le desservant.

L'apport essentiel de la décision Commune des Saintes-Maries-de-la-Mer consiste ainsi, s'agissant de l'utilisation culturelle et touristique de l'édifice cultuel, à limiter l'autorisation obligatoire du desservant à l'accès aux aménagements et dépendances de l'édifice affectés au culte, cette affectation pouvant être directe (utilisation de la dépendance ou de l'aménagement eux-mêmes à des fins cultuelles) ou indirecte (nécessité d'emprunter ces aménagements et dépendance pour accéder aux parties de l'édifice affectées au culte). En excluant l'autorisation obligatoire pour la visite de parties non cultuelles, et donc pour l'usage culturel et touristique d'aménagements et dépendances non cultuels, la solution retenue préserve « en creux » le principe de l'autorisation obligatoire pour la visite de parties cultuelles, et donc pour l'usage culturel 
et touristique d'aménagements et dépendances cultuels. Enfin, l'appréciation de la compatibilité entre les visites touristiques et les célébrations religieuses, donc de la compatibilité de l'utilisation avec l'affectation cultuelle, relève en effet désormais du contrôle a posteriori du juge (à moins peut-être que le desservant ne saisisse le juge d'un référé-suspension dirigé contre la décision du maire refusant d'annuler les visites les jours de célébration) et non plus de l'accord a priori de l'affectataire cultuel. L'appréciation de la compatibilité entre les visites touristiques et l'utilisation cultuelle de l'édifice et de ses aménagements et dépendances n'est plus le fruit d'un dialogue entre la collectivité propriétaire de l'édifice et l'affectataire, mais le résultat d'une analyse effectuée par cette dernière sous le contrôle du juge ${ }^{52}$.

$\mathrm{Au}$ total, la décision Commune des Saintes-Maries-de-la-Mer consacre donc une première hypothèse d'utilisation culturelle et touristique d'une partie de l'édifice cultuel sans l'accord du desservant, hypothèse qui devrait cependant rester isolée et peu appliquée, dès lors que la plupart des utilisations culturelles des édifices cultuels (en particulier de ceux classés ou inscrits au titre des monuments historiques) consistent en des visites de l'intérieur de l'édifice dont les modalités d'organisation par la collectivité propriétaire doivent cette fois recueillir l'accord préalable du desservant. Par ailleurs, l'application de la jurisprudence Commune des Saintes-Maries-de-la-Mer devrait être limitée aux dépendances et aménagements de l'édifice cultuel accessibles par l'extérieur de cet édifice.

\section{CONCLUSION}

L'utilisation de l'édifice cultuel à des fins non cultuelles, dès lors qu'elle doit être compatible avec l'affectation cultuelle dont est maître et gardien l'affectataire cultuel, pose la question non seulement de l'autorité chargée de vérifier et garantir cette compatibilité (il s'agira très généralement de l'affectataire mais il pourra s'agir résiduellement du propriétaire), mais aussi et surtout celle des moyens juridictionnels de la faire respecter. La jurisprudence administrative montre sur ce point que l'affectataire dispose de plusieurs

52. Le Conseil d'État estime en l'espèce qu'« il ne ressort pas des pièces du dossier que les modalités d'organisation de ces visites touristiques conduiraient à perturber l'exercice du culte à l'intérieur de l'église ou seraient incompatibles avec l'affectation de l'église sur le toit de laquelle les aménagements visités sont situés [et] qu'en particulier, il ne ressort pas des pièces du dossier que ces modalités d'organisation perturberaient la fréquentation à des fins cultuelles de la chapelle Saint-Michel ». 
recours pour contester une utilisation non cultuelle de l'édifice dont il a la charge et pour laquelle il n'a pas donné son consentement, mais l'on peut se demander ce qui se passerait si l'affectataire donnait son consentement à une telle utilisation contre l'avis de la hiérarchie religieuse. Le « légitimisme religieux » du juge administratif incite à penser qu'il ferait prévaloir l'opposition de la hiérarchie sur l'accord de l'affectataire.

Enfin, l'on assistera probablement à une contractualisation de l'accord de l'affectataire, qui semble la mieux à même de prévoir les conditions et modalités (tant matérielles que financières) de l'utilisation de l'édifice à des fins non cultuelles ainsi que les engagements réciproques de l'affectataire et du propriétaire pour les mettre en œuvre. L'on peut donc s'attendre à la naissance d'un contentieux contractuel cultuel, qui devrait également ressortir de la compétence du juge administratif puisque le contrat en cause aurait pour objet l'utilisation du domaine public cultuel. 